\title{
Effect of Various Modules against Leaf Hopper, Amrasca biguttula biguttula Ishida on Okra
}

\author{
Vandana D. Mohod*, A.Y. Thakare, A.V. Kolhe and D.B. Undirwade
}

Department of Entomology, Dr. Panjabrao Deshmukh Krishi Vidyapeeth Akola, India

*Corresponding author

\begin{tabular}{|l|}
\hline Ke y w o r d s \\
Okra, Leaf hopper, \\
Module \\
\hline Article Info \\
\hline Accepted: \\
12 June 2019 \\
Available Online: \\
10 July 2019 \\
\hline
\end{tabular}

\section{A B S T R A C T}

Field studies were undertaken to assess the Effect of various modules against leaf hopper, Amrasca biguttula biguttula Ishida on Okra of eight modules against leaf hopper during summer 2014-15 and kharif, 2015-16 at Chilli and Vegetable Research Unit, Dr. Panjabrao Deshmukh Krishi Vidyapeeth, Akola (M.S.). Among eight different modules tested the lowest population of leaf hopper (5.07/leaf) was recorded in M6, followed by module M7 (5.31LH/leaf) and M5 (5.79LH/leaf) and these treatments were found statistically at par among themselves and superior over rest of the treatments during summer 2014-15. Similar trend was observed during kharif 2015-16 indicating minimum population of leaf hopper was observed in module M6 (4.57/leaf), followed by module M7 (4.74/leaf) and module M5 (5.19/leaf). The lowest population of leaf hopper (4.82/leaf) was recorded in module M6 followed by M7 (5.02LH/leaf) and M5 (5.49LH/leaf) and they were statistically at par with other. Module M6 and M7 were significantly superior over remaining treatments except module M5 which was further found to be statistically at par with M4 $(6.57 \mathrm{LH} / \mathrm{leaf})$ and M2 (6.69LH/leaf) and superior over M3, M1 and M8 during summer and kharif season, respectively

\section{Introduction}

Okra (Abelmoschus esculentus L. Monech) or Bhindi is an important vegetable crop due to its nutritional value. It is good source of vitamin $\mathrm{A}, \mathrm{B}, \mathrm{C}$ and is also rich in protein, minerals and iodine (Baloch et al., 1990). It has good nutritional value, particularly the high content of vitamin C $(30 \mathrm{mg} / 100 \mathrm{~g})$, calcium $(90 \mathrm{mg} / 100 \mathrm{~g})$, iron $(1.5 \mathrm{mg} / 100 \mathrm{~g})$ and other minerals like magnesium and potassium, fats and carbohydrates (Aykroud, 1963). Among the commercially cultivated vegetable crops in India, okra [Abelmoschus esculentus (L.) Moench] ranked sixth in terms of area and production with country contributing about 73.25 per cent to the world's total production (Anon., 2013). The crop is popular worldwide for its nutritionally rich tender fruits, which are consumed as raw or cooked. Besides, its use as food, the pods and seeds are used for the manufacture of 
paper and biodiesel (Anwar, 2010). The major okra growing states Maharashtra (Anon., 2012). The continuous growth is congenial for the infestation of insect pests and it is one of the major limiting factors in the profitable cultivation of the crop (Ashok Kumar, et al., 2009). Many insect pests incidence were recorded from sowing upto harvest on bhendi plants in India and listed the most destructive insect pests as leafhopper, Amrasca biguttula biguttula (Ishida), aphid, Aphis gossypii (Glover), whiteflies, Bemisia tabaci (Gennadius), fruit borer, Helicoverpa armigera (Hubn.), spotted bollworm, Earias vittella (Fabricius) and Earias insulana (Boisd.) (Mane, et al., 2010). Among these aphids, jassids and whitefly are considered as important sucking insect pests of okra. Krishnaiah (1980) reported about 40-56 per cent losses in yield of okra due to leafhoppers.

The demand of vegetables like Okra increases day by day. Being short duration and high yielding, growers get more profit per unit area. However, various problems are faced by cultivators at the time when incidence of sucking pests observed in initial stages on Okra crop. Boosting of its production is of prime importance and considerable.

To tackle this sucking pest menace, a number of chemical insecticides are liberally sprayed on this vegetable crop, which led to several problems like toxic residues, elimination of natural enemies, environmental disharmony and development of resistance.

Due to the presence of pesticidal residues in the final commodity, there is a risk of rejection of whole consignments during export. Investigations were carried out in order to find out suitable, effective, ecofriendly and economical plant protection measures against major sucking pests of Okra with the objectives. To find out effective module against leaf hopper.

\section{Materials and Methods}

Evaluation of various modules against leaf hopper on okra with trials were conducted during summer 2014-15 and Kharif 2015-16 at Chilli and Vegetable, Research Unit, Dr. PDKV., Akola. Okra variety Akola bahar sown in well prepared land and the crop was grown following all standard package of practices. The experiments were laid in Randomized Block Design (RBD) with three replications and eight modules. The crop was sown at a spacing of $45 \mathrm{~cm} \mathrm{x} 30 \mathrm{~cm}$ and $60 \mathrm{cmx}$ $45 \mathrm{~cm}$ with gross plot size of $4.95 \mathrm{~m} \mathrm{x} 3 \mathrm{~m}$. and $5.1 \mathrm{mx} 3.0 \mathrm{~m}$. Treatment schedule was as follows.

The experiment was conducted in RBD with eight modules replicated thrice. Pre-treatment observation was taken $24 \mathrm{hrs}$ before spray and post treatment observation was recorded at 3 , 7 and 14 days after each spraying on randomly selected 5 plants per plot. For observation on nymphal leafhopper, was recorded on 3 leaves (top, middle and bottom canopy of the plant) per plant at 3, 7 and 14 DAS on five randomly selected plants per plot after each spraying. Data so obtained during summer 2014-15 and kharif 2015-16 were statistically analysed after suitable transformation and the inferences were drawn basing on the results.

\section{Results and Discussion}

The data presented in Table 1 showed that data on the incidence of aphid 24 hours before first spray during summer 2014-15 were found statistically non-significant. However, incidence of leaf hopper in various treatments was found in the range of 0.07 leaf hopper/leaf in module M6 to 0.60 leaf hopper/leaf in module M8. Mean Leaf hopper population at 3 days after spray module M6 had recorded the minimum population of leaf hopper (3.27LH/leaf) followed by M7 (3.36LH/leaf), M5 (3.66LH/leaf) and M4 (4.36LH/leaf) and 
they were found statistically at par among themselves and superior over rest of the modules. Treatment module M3 (4.54LH/leaf) and M2 (4.53LH/leaf) were statistically equal effective with M7, M5, M4. The treatment M1 (5.19 LH/leaf) had exerted statistically similar effect to those of treated module M4 and M3 and M2. The unprotected module (M8) had registered the maximum population of leaf hopper (6.88/leaf).

Mean Leaf hopper population at 7 days after spray the module M7 (4.16LH/leaf) had shown the least population of leaf hopper followed by M6 (4.56LH/leaf) and M3 (5.56LH/leaf) and they were statistically at par with each and superior over rest of the modules. Module M6 was further found statistically similar with M3 (5.56 LH/leaf) M5 (5.83 LH/leaf), M2 (6.03 LH/leaf) and M4 (6.09 LH/leaf). The treatment model M4 was further statistically at par with M1 (7.91LH/leaf). The untreated module M8 had accounted the highest population of leaf hopper (11.33 LH/leaf). The minimum population of leaf hopper at 14 days after spray was registered in M6 (7.40/leaf) followed by M5 (7.86/leaf), M7 (8.39/leaf) and M2 (9.15/leaf) and these treatments were found statistically similar effective. Treatment module M7 and module M2 were found further statistically at par with M4 (10.84/leaf), M1 (11.05/leaf) and M3 (11.28/leaf) and superior over (M8). The highest population of leaf hopper (16.12/leaf) was noticed in unprotected control (M8).The data of cumulative mean of leaf hopper population during summer $2014-15$ the lowest population of leaf hopper (5.07/leaf) was recorded in M6, followed by module M7 (5.31LH/leaf) and M5 (5.79LH/leaf) and these treatments were found statistically at par among themselves and superior over rest of the treatments. The treatment module M2 (6.57LH/leaf) had also shown the statistically similar effectiveness with earlier treatment modules M7 and M5 and this module.M2 was further found to be statistically at par with module M4 (7.09/leaf) M3 (7.13/leaf) and M1 (8.05/leaf). The untreated module M8 had registered the maximum population of leaf hopper (11.44/leaf).

The data presented in Table 2 showed that data on the incidence of aphid 24 hours before first spray during kharif 2015-16 were found statistically non-significant. However, incidence of leaf hopper in various treatments was found in the range of 0.07 leaf hopper /leaf in module M7 to 0.17 leaf hopper/leaf in module M8.

The data as regard to response of various treatment modules on the leaf hopper population during kharif 2015- 16 at 3 days after spray that the result was found statistically significant. All the treated modules were significantly superior over untreated module. The treatment module M7 had recorded the lowest population of leaf hopper (1.41LH/leaf), followed by model M6 (1.62 LH /leaf) and they were found statistically at par with each other and both the treatments appeared to be statistically superior over the rest of the treatments. The treatment modules M5, M3, M4 and M2 were statistically similar effective showing the population of leaf hopper to the extent of 2.63, $2.84,2.87$ and 3.36 leaf hopper/leaf, respectively and superior over module $\mathrm{M} 1$ and M8. Of these, the later module (M2) was further found statistically at par with M1 (4.27 LH /leaf). The highest population of leaf hopper was recorded in unprotected module M8 (12.35 LH /leaf). At 7 days after spray it is seen that the treatment module M6 had accounted the minimum population of leaf hopper (4.45/leaf), and was found statistically at par with the treatment modules M7(4.90 LH /leaf), Module M5 (5.16 LH /leaf) and module M4 (5.84 LH /leaf). 


\begin{tabular}{|c|c|}
\hline \multicolumn{2}{|c|}{ Treatments details } \\
\hline $\mathbf{M}_{1}$ & $\begin{array}{l}\text { - Soil application of neem cake @ } 250 \mathrm{~kg} / \mathrm{ha} \text { at time of sowing } \\
\text { - Installation of yellow sticky trap at } 15 \mathrm{DAS}(30 \mathrm{x} 15 \mathrm{~cm} \text { size of foam sheet) at } 2 \text { opposite corner along crop } \\
\text { canopy and } 15 \mathrm{~cm} \text { above crop canopy } \\
\text { - Weekly clipping of infested shoots from the appearance of pests. } \\
\text { - Foliar application of azadirachtin } 1 \% \mathrm{w} / \mathrm{w} @ 5 \mathrm{ml} / \mathrm{L} \text { at } 15 \text { days interval from sowing to } 75 \text { days after sowing. }\end{array}$ \\
\hline $\mathbf{M}_{2}$ & 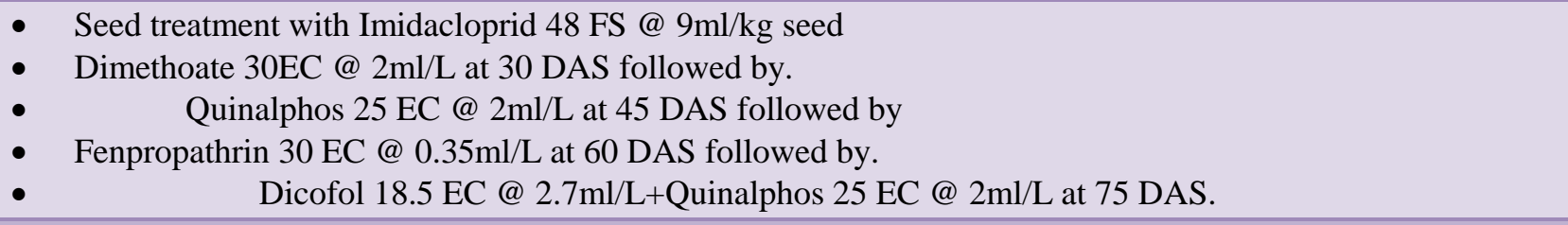 \\
\hline $\mathbf{M}_{3}$ & 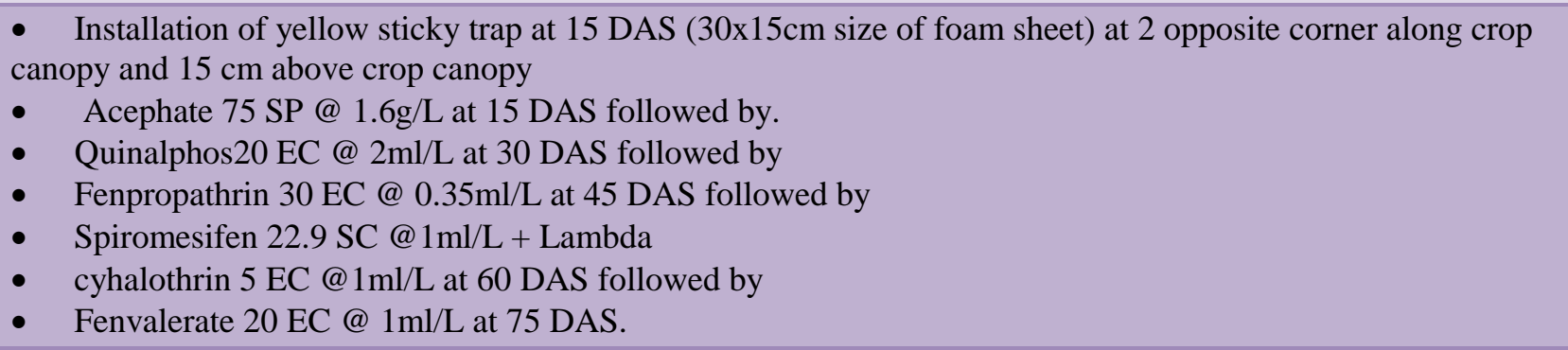 \\
\hline $\mathbf{M}_{4}$ & $\begin{array}{ll}\text { - } & \text { Triazophos } 40 \mathrm{EC} @ 2 \mathrm{ml} / \mathrm{L} \text { at } 15 \mathrm{DAS} \text { followed by } \\
\text { - } & \text { Fenvalerate 20EC @ } 1 \mathrm{ml} / \mathrm{L} \text { at } 30 \mathrm{DAS} \text { followed by } \\
\text { - } & \text { Quinalphos } 25 \mathrm{EC} @ 2 \mathrm{ml} / \mathrm{L}+\text { Spiromesifen } 22.9 \mathrm{SC} @ 1 \mathrm{ml} / \mathrm{Lat} 45 \mathrm{DAS} \text { followed by } \\
\text { - } & \text { Fenpropathrin } 30 \mathrm{EC} @ 0.35 \mathrm{ml} / \mathrm{L} \text { at } 60 \mathrm{DAS} \text { followed by } \\
\text { - } & \text { Lambda cyhalothrin } 5 \mathrm{EC} @ 1 \mathrm{ml} / \mathrm{L} \text { at } 75 \mathrm{DAS} \text {. }\end{array}$ \\
\hline $\mathbf{M}_{5}$ & $\begin{array}{l}\text { - Soil application of neem cake @250kg/ha at time of sowing } \\
\text { - Installation of yellow sticky trap at } 15 \text { DAS }(30 \mathrm{x} 15 \mathrm{~cm} \text { size of foam sheet) at } 2 \text { opposite corner along crop } \\
\text { canopy and } 15 \mathrm{~cm} \text { above crop canopy } \\
\text { - Thiamethoxam } 25 \mathrm{WG} @ 0.2 \mathrm{~g} / \mathrm{L} \text { at } 30 \text { DAS followed by } \\
\text { - } \quad \text { Fenpropathrin } 30 \mathrm{EC} @ 0.35 \mathrm{ml} / \mathrm{L} \text { at } 45 \mathrm{DAS} \text { followed by } \\
\text { - } \quad \text { Lambda cyhalothrin } 5 \mathrm{EC} @ 1 \mathrm{ml} / \mathrm{L} \text { at } 60 \mathrm{DAS} \text { followed by } \\
\text { - } \quad \text { Triazophos } 40 \mathrm{EC} @ 2 \mathrm{ml} / \mathrm{L}+\mathrm{Dicofol} 18.5 \mathrm{EC} @ 2.7 \mathrm{ml} / \mathrm{L} \text { at } 75 \mathrm{DAS} \text {. }\end{array}$ \\
\hline $\mathbf{M}_{6}$ & $\begin{array}{l}\text { - Seed treatment with imidacloprid } 48 \mathrm{FS} @ 9 \mathrm{ml} / \mathrm{kg} \text { seed } \\
\text { - Soil application neem cake @ } 250 \mathrm{~kg} / \mathrm{ha} \text { at time of sowing } \\
\text { - Installation of yellow sticky trap at } 15 \mathrm{DAS}(30 \mathrm{x} 15 \mathrm{~cm} \text { size of foam sheet) at } 2 \text { opposite corner along crop } \\
\text { canopy and } 15 \mathrm{~cm} \text { above crop canopy } \\
\text { - } \quad \text { Cypermethrin } 25 \mathrm{EC} @ 0.4 \mathrm{ml} \text { at } 30 \mathrm{DAS} \text { followed by } \\
\text { - } \quad \text { Triazophos } 40 \mathrm{EC} @ 2 \mathrm{ml} / \mathrm{L} \text { at } 45 \mathrm{DAS} \text { followed by } \\
\text { - } \quad \text { Fenpropathrin } 30 \mathrm{EC} @ 0.35 \mathrm{ml} / \mathrm{L} \text { at } 60 \mathrm{DAS} \text { followed by } \\
\text { - } \quad \text { Acephate } 75 \mathrm{SP} @ 1.6 \mathrm{~g} / \mathrm{L}+\mathrm{Sppiromesifen} 22.9 \mathrm{SC} @ 1 \mathrm{ml} / \mathrm{Lat} 75 \mathrm{DAS} .\end{array}$ \\
\hline $\mathbf{M}_{7}$ &  \\
\hline $\mathbf{M}_{8}$ & Untreated control \\
\hline
\end{tabular}


Table.1 Effect of various modules on cumulative mean population of leaf hopper on okra during summer 2014-15

\begin{tabular}{|c|c|c|c|c|c|c|}
\hline \multirow[t]{2}{*}{$\begin{array}{l}\text { Tr. } \\
\text { No. }\end{array}$} & \multirow[t]{2}{*}{ Treatment details } & \multicolumn{4}{|c|}{$\begin{array}{l}\text { Cum. Mean Leaf hopper } \\
\text { population / leaf }\end{array}$} & \multirow{2}{*}{$\begin{array}{c}\begin{array}{c}\text { Cum. } \\
\text { pooled } \\
\text { mean }\end{array} \\
\text { Based on } \\
\text { five spray }\end{array}$} \\
\hline & & $\begin{array}{c}\text { Pre- } \\
\text { treat.obs. } \\
\text { Before } 24 \\
\text { hrs }\end{array}$ & $\begin{array}{c}3 \\
\text { DAT }\end{array}$ & $\begin{array}{c}7 \\
\text { DAT }\end{array}$ & $\begin{array}{c}14 \\
\text { DAT }\end{array}$ & \\
\hline M1 & SA.YST, Azadirachtin 1\%w/w & $\begin{array}{c}0.20 \\
(0.82)\end{array}$ & $\begin{array}{c}5.19 \\
(2.28)\end{array}$ & $\begin{array}{c}7.91 \\
(2.81)\end{array}$ & $\begin{array}{l}11.05 \\
(3.32)\end{array}$ & $\begin{array}{c}8.05 \\
(2.84)\end{array}$ \\
\hline M2 & $\begin{array}{l}\text { ST, Dim, Quin, Fenpo, } \\
\text { Dico+Quin. }\end{array}$ & $\begin{array}{c}0.13 \\
(0.79)\end{array}$ & $\begin{array}{c}4.53 \\
(2.12)\end{array}$ & $\begin{array}{c}6.03 \\
(2.45)\end{array}$ & $\begin{array}{c}9.15 \\
(3.02)\end{array}$ & $\begin{array}{c}6.57 \\
(2.56)\end{array}$ \\
\hline M3 & $\begin{array}{l}\text { YST, Ace, Quin Fenpo, Spi+Lam, } \\
\text { Fenv. }\end{array}$ & $\begin{array}{c}0.33 \\
(0.90)\end{array}$ & $\begin{array}{c}4.54 \\
(2.12)\end{array}$ & $\begin{array}{l}5.56 \\
(2.36)\end{array}$ & $\begin{array}{l}11.28 \\
(3.36)\end{array}$ & $\begin{array}{l}7.13 \\
(2.67)\end{array}$ \\
\hline M4 & $\begin{array}{l}\text { Tria, Fenv, Quin+Spir, Fenpo, } \\
\text { Lamb }\end{array}$ & $\begin{array}{c}0.33 \\
(0.89)\end{array}$ & $\begin{array}{l}4.36 \\
(2.09)\end{array}$ & $\begin{array}{c}6.09 \\
(2.47)\end{array}$ & $\begin{array}{l}10.84 \\
(3.28)\end{array}$ & $\begin{array}{c}7.09 \\
(2.66)\end{array}$ \\
\hline M5 & $\begin{array}{l}\text { SA, YST, Thia, Fenpo, Lamb, } \\
\text { Tria+Dico }\end{array}$ & $\begin{array}{c}0.33 \\
(0.91)\end{array}$ & $\begin{array}{c}3.66 \\
(1.91)\end{array}$ & $\begin{array}{c}5.83 \\
(2.41)\end{array}$ & $\begin{array}{c}7.86 \\
(2.80)\end{array}$ & $\begin{array}{c}5.79 \\
(2.41)\end{array}$ \\
\hline M6 & $\begin{array}{l}\text { ST, SA, YST, Cyper, Tria, Fenpo, } \\
\text { Ace+Spir }\end{array}$ & $\begin{array}{c}0.07 \\
(0.75)\end{array}$ & $\begin{array}{c}3.27 \\
(1.80)\end{array}$ & $\begin{array}{c}4.56 \\
(2.12)\end{array}$ & $\begin{array}{c}7.40 \\
(2.71)\end{array}$ & $\begin{array}{c}5.07 \\
(2.25)\end{array}$ \\
\hline M7 & $\begin{array}{l}\text { ST, SA, YST, Cyper+Dim, Fenpo, } \\
\text { Ethion+Quin, Lamb. }\end{array}$ & $\begin{array}{c}0.13 \\
(0.79)\end{array}$ & $\begin{array}{c}3.36 \\
(1.83)\end{array}$ & $\begin{array}{c}4.16 \\
(2.02)\end{array}$ & $\begin{array}{c}8.39 \\
(2.90)\end{array}$ & $\begin{array}{c}5.31 \\
(2.30)\end{array}$ \\
\hline M8 & Untreated control & $\begin{array}{c}0.60 \\
(1.02)\end{array}$ & $\begin{array}{c}6.88 \\
(2.61)\end{array}$ & $\begin{array}{l}11.33 \\
(3.36)\end{array}$ & $\begin{array}{l}16.12 \\
(3.99)\end{array}$ & $\begin{array}{l}11.44 \\
(3.37)\end{array}$ \\
\hline & $\mathrm{SE}(\mathrm{m}) \pm$ & 0.09 & 0.10 & 0.11 & 0.17 & 0.09 \\
\hline & $\mathrm{CD}$ at $5 \%$ & NS & 0.30 & 0.35 & 0.51 & 0.28 \\
\hline & $\mathrm{CV} \%$ & 18.70 & 8.25 & 8.01 & 9.17 & 6.11 \\
\hline
\end{tabular}

N.B: Figures in parenthesis are square root transformation 
Table.2 Effect of various modules on cumulative mean population of leaf hopper on okra during kharif 2015-16

\begin{tabular}{|c|c|c|c|c|c|c|}
\hline \multirow[t]{2}{*}{$\begin{array}{l}\text { Tr. } \\
\text { No. }\end{array}$} & \multirow[t]{2}{*}{ Treatment details } & \multirow{2}{*}{$\begin{array}{c}\text { Pre- } \\
\text { tret.obs. } \\
\text { before } \\
24 \text { hrs }\end{array}$} & \multicolumn{3}{|c|}{$\begin{array}{l}\text { Cum. Mean Leaf hopper } \\
\text { population / leaf }\end{array}$} & \multirow{2}{*}{$\begin{array}{c}\begin{array}{c}\text { Pooled } \\
\text { mean }\end{array} \\
\begin{array}{c}\text { Based } \\
\text { on five } \\
\text { spray }\end{array}\end{array}$} \\
\hline & & & $\begin{array}{c}3 \\
\text { DAT }\end{array}$ & $\begin{array}{c}7 \\
\text { DAT }\end{array}$ & $\begin{array}{c}14 \\
\text { DAT }\end{array}$ & \\
\hline M1 & SA.YST, Azadirachtin 1\%w/w & $\begin{array}{c}0.13 \\
(0.79)\end{array}$ & $\begin{array}{c}4.27 \\
(2.06)\end{array}$ & $\begin{array}{c}9.51 \\
(3.08)\end{array}$ & $\begin{array}{l}11.22 \\
(3.35)\end{array}$ & $\begin{array}{c}8.34 \\
(2.89)\end{array}$ \\
\hline M2 & ST, Dim, Quin, Fenpo, Dico+Quin. & $\begin{array}{c}0.07 \\
(0.75)\end{array}$ & $\begin{array}{c}3.36 \\
(1.83)\end{array}$ & $\begin{array}{c}7.21 \\
(2.68)\end{array}$ & $\begin{array}{c}9.86 \\
(3.14)\end{array}$ & $\begin{array}{l}6.81 \\
(2.61)\end{array}$ \\
\hline M3 & $\begin{array}{l}\text { YST, Ace, Quin Fenpo, Spi+Lam, } \\
\text { Fenv. }\end{array}$ & $\begin{array}{l}0.12 \\
(0.78\end{array}$ & $\begin{array}{c}2.84 \\
(1.68)\end{array}$ & $\begin{array}{l}7.36 \\
(2.70)\end{array}$ & $\begin{array}{c}9.79 \\
(3.13)\end{array}$ & $\begin{array}{l}6.66 \\
(2.58)\end{array}$ \\
\hline M4 & Tria, Fenv, Quin+Spir, Fenpo, Lamb & $\begin{array}{c}0.16 \\
(0.81)\end{array}$ & $\begin{array}{c}2.87 \\
(1.69)\end{array}$ & $\begin{array}{c}5.84 \\
(2.41)\end{array}$ & $\begin{array}{c}9.41 \\
(3.07)\end{array}$ & $\begin{array}{c}6.04 \\
(2.46)\end{array}$ \\
\hline M5 & $\begin{array}{l}\text { SA, YST, Thia, Fenpo, Lamb, } \\
\text { Tria+Dico }\end{array}$ & $\begin{array}{c}0.13 \\
(0.79)\end{array}$ & $\begin{array}{c}2.63 \\
(1.62)\end{array}$ & $\begin{array}{l}5.16 \\
(2.27)\end{array}$ & $\begin{array}{l}7.78 \\
(2.78)\end{array}$ & $\begin{array}{c}5.19 \\
(2.28)\end{array}$ \\
\hline M6 & $\begin{array}{l}\text { ST, SA, YST, Cyper, Tria, Fenpo, } \\
\text { Ace+Spir }\end{array}$ & $\begin{array}{c}0.08 \\
(0.76)\end{array}$ & $\begin{array}{c}1.62 \\
(1.27)\end{array}$ & $\begin{array}{l}4.45 \\
(2.11)\end{array}$ & $\begin{array}{l}7.65 \\
(2.76)\end{array}$ & $\begin{array}{c}4.57 \\
(2.14)\end{array}$ \\
\hline M7 & $\begin{array}{l}\text { ST, SA, YST, Cyper+Dim, Fenpo, } \\
\text { Ethion+Quin, Lamb. }\end{array}$ & $\begin{array}{c}0.07 \\
(0.75)\end{array}$ & $\begin{array}{c}1.41 \\
(1.19)\end{array}$ & $\begin{array}{l}4.90 \\
(2.21)\end{array}$ & $\begin{array}{c}7.91 \\
(2.81)\end{array}$ & $\begin{array}{c}4.74 \\
(2.18)\end{array}$ \\
\hline M8 & Untreated control & $\begin{array}{c}0.17 \\
(0.81)\end{array}$ & $\begin{array}{l}12.35 \\
(3.50)\end{array}$ & $\begin{array}{l}15.48 \\
(3.93)\end{array}$ & $\begin{array}{l}18.47 \\
(4.28)\end{array}$ & $\begin{array}{l}15.44 \\
(3.92)\end{array}$ \\
\hline & $\mathrm{SE}(\mathrm{m}) \pm$ & 0.03 & 0.08 & 0.10 & 0.13 & 0.09 \\
\hline & $\mathrm{CD}$ at $5 \%$ & NS & 0.26 & 0.32 & 0.41 & 0.28 \\
\hline & $\mathrm{CV} \%$ & 7.31 & 7.92 & 7.74 & 7.33 & 6.10 \\
\hline
\end{tabular}

N.B: Figures in parenthesis are square root transformation 
Table.3 Effect of various modules on comparative population of leaf hopper on okra during summer 2014-15 and kharif season 2015-16

\begin{tabular}{|c|c|c|c|c|c|c|c|c|c|c|c|c|c|c|}
\hline \multirow{2}{*}{$\begin{array}{l}\text { Tr. } \\
\text { No. }\end{array}$} & \multirow[t]{2}{*}{ Treatment details } & \multirow{2}{*}{$\begin{array}{c}\text { Pre- } \\
\text { treat.obs } \\
.24 \text { hrs. } \\
\text { before }\end{array}$} & \multicolumn{3}{|c|}{3 DAT } & \multicolumn{3}{|c|}{7 DAT } & \multicolumn{3}{|c|}{14 DAT } & \multicolumn{3}{|c|}{ Pooled mean } \\
\hline & & & Summer & Kharif & $\begin{array}{c}\text { Poole } \\
\text { d } \\
\text { Mean }\end{array}$ & $\begin{array}{c}\text { Sum } \\
\text { mer }\end{array}$ & $\begin{array}{c}\text { Khari } \\
\text { f }\end{array}$ & $\begin{array}{c}\text { Poole } \\
\text { d } \\
\text { Mean }\end{array}$ & $\begin{array}{c}\text { Summ } \\
\text { er }\end{array}$ & $\begin{array}{c}\text { Khari } \\
\text { f }\end{array}$ & $\begin{array}{c}\text { Pooled } \\
\text { Mean }\end{array}$ & $\begin{array}{c}\text { Summe } \\
\mathbf{r}\end{array}$ & Kharif & $\begin{array}{c}\text { Pooled } \\
\text { Mean }\end{array}$ \\
\hline M1 & SA.YST, Azadirachtin $1 \% \mathrm{w} / \mathrm{w}$ & $\begin{array}{c}0.16 \\
(0.81)\end{array}$ & $\begin{array}{c}5.19 \\
(2.28)\end{array}$ & $\begin{array}{c}4.27 \\
(2.06)\end{array}$ & $\begin{array}{c}4.73 \\
(2.17)\end{array}$ & $\begin{array}{c}7.91 \\
(2.81)\end{array}$ & $\begin{array}{c}9.51 \\
(3.08)\end{array}$ & $\begin{array}{c}8.71 \\
(2.95)\end{array}$ & $\begin{array}{l}11.05 \\
(3.32)\end{array}$ & $\begin{array}{l}11.22 \\
(3.35)\end{array}$ & $\begin{array}{l}11.13 \\
(3.34)\end{array}$ & $\begin{array}{c}8.05 \\
(2.84)\end{array}$ & $\begin{array}{c}8.34 \\
(2.89)\end{array}$ & $\begin{array}{c}8.19 \\
(2.86)\end{array}$ \\
\hline M2 & ST, Dim, Quin, Fenpo, Dico+Quin. & $\begin{array}{c}0.10 \\
(0.77)\end{array}$ & $\begin{array}{c}4.53 \\
(2.12)\end{array}$ & $\begin{array}{c}3.36 \\
(1.83)\end{array}$ & $\begin{array}{c}3.95 \\
(1.98)\end{array}$ & $\begin{array}{c}6.03 \\
(2.45)\end{array}$ & $\begin{array}{c}7.21 \\
(2.68)\end{array}$ & $\begin{array}{c}6.62 \\
(2.57)\end{array}$ & $\begin{array}{c}9.15 \\
(3.02)\end{array}$ & $\begin{array}{c}9.86 \\
(3.14)\end{array}$ & $\begin{array}{c}9.51 \\
(3.08)\end{array}$ & $\begin{array}{c}6.57 \\
(2.56)\end{array}$ & $\begin{array}{c}6.81 \\
(2.61)\end{array}$ & $\begin{array}{c}6.69 \\
(2.59)\end{array}$ \\
\hline M3 & $\begin{array}{l}\text { YST, Ace, Quin Fenpo, Spi+Lam, } \\
\text { Fenv. }\end{array}$ & $\begin{array}{c}0.23 \\
(0.84)\end{array}$ & $\begin{array}{c}4.54 \\
(2.12)\end{array}$ & $\begin{array}{c}2.84 \\
(1.68)\end{array}$ & $\begin{array}{c}3.69 \\
(1.90)\end{array}$ & $\begin{array}{c}5.56 \\
(2.36)\end{array}$ & $\begin{array}{l}7.36 \\
(2.70)\end{array}$ & $\begin{array}{c}6.46 \\
(2.53)\end{array}$ & $\begin{array}{l}11.28 \\
(3.36)\end{array}$ & $\begin{array}{c}9.79 \\
(3.13)\end{array}$ & $\begin{array}{l}10.54 \\
(3.24)\end{array}$ & $\begin{array}{l}7.13 \\
(2.67)\end{array}$ & $\begin{array}{c}6.66 \\
(2.58)\end{array}$ & $\begin{array}{c}6.90 \\
(2.62)\end{array}$ \\
\hline M4 & Tria, Fenv, Quin+Spir, Fenpo, Lamb & $\begin{array}{c}0.25 \\
(0.85)\end{array}$ & $\begin{array}{l}4.36 \\
(2.09)\end{array}$ & $\begin{array}{c}2.87 \\
(1.69)\end{array}$ & $\begin{array}{c}3.61 \\
(1.89)\end{array}$ & $\begin{array}{c}6.09 \\
(2.47)\end{array}$ & $\begin{array}{c}5.84 \\
(2.41)\end{array}$ & $\begin{array}{l}5.96 \\
(2.44)\end{array}$ & $\begin{array}{l}10.84 \\
(3.28)\end{array}$ & $\begin{array}{c}9.41 \\
(3.07)\end{array}$ & $\begin{array}{l}10.12 \\
(3.18)\end{array}$ & $\begin{array}{c}7.12 \\
(2.66)\end{array}$ & $\begin{array}{c}6.04 \\
(2.46)\end{array}$ & $\begin{array}{c}6.57 \\
(2.56)\end{array}$ \\
\hline M5 & $\begin{array}{l}\text { SA, YST, Thia, Fenpo, Lamb, } \\
\text { Tria+Dico }\end{array}$ & $\begin{array}{c}0.23 \\
(0.85)\end{array}$ & $\begin{array}{c}3.66 \\
(1.91)\end{array}$ & $\begin{array}{c}2.63 \\
(1.62)\end{array}$ & $\begin{array}{c}3.15 \\
(1.77)\end{array}$ & $\begin{array}{c}5.83 \\
(2.41)\end{array}$ & $\begin{array}{c}5.16 \\
(2.27)\end{array}$ & $\begin{array}{c}5.49 \\
(2.34)\end{array}$ & $\begin{array}{l}7.86 \\
(2.80)\end{array}$ & $\begin{array}{l}7.78 \\
(2.78)\end{array}$ & $\begin{array}{c}7.82 \\
(2.79)\end{array}$ & $\begin{array}{l}5.79 \\
(2.41)\end{array}$ & $\begin{array}{c}5.19 \\
(2.28)\end{array}$ & $\begin{array}{l}5.49 \\
(2.34)\end{array}$ \\
\hline M6 & $\begin{array}{l}\text { ST, SA, YST, Cyper, Tria, Fenpo, } \\
\text { Ace+Spir }\end{array}$ & $\begin{array}{c}0.08 \\
(0.76)\end{array}$ & $\begin{array}{c}3.27 \\
(1.80)\end{array}$ & $\begin{array}{c}1.62 \\
(1.27)\end{array}$ & $\begin{array}{c}2.45 \\
(1.54)\end{array}$ & $\begin{array}{c}4.56 \\
(2.12)\end{array}$ & $\begin{array}{c}4.45 \\
(2.11)\end{array}$ & $\begin{array}{c}4.50 \\
(2.11)\end{array}$ & $\begin{array}{l}7.40 \\
(2.71)\end{array}$ & $\begin{array}{c}7.65 \\
(2.76)\end{array}$ & $\begin{array}{c}7.52 \\
(2.74)\end{array}$ & $\begin{array}{c}5.07 \\
(2.25)\end{array}$ & $\begin{array}{c}4.57 \\
(2.14)\end{array}$ & $\begin{array}{c}4.82 \\
(2.19)\end{array}$ \\
\hline M7 & $\begin{array}{l}\text { ST, SA, YST, Cyper+Dim, Fenpo, } \\
\text { Ethion+Quin, Lamb. }\end{array}$ & $\begin{array}{c}0.10 \\
(0.77)\end{array}$ & $\begin{array}{c}3.36 \\
(1.83)\end{array}$ & $\begin{array}{c}1.41 \\
(1.19)\end{array}$ & $\begin{array}{c}2.39 \\
(1.51)\end{array}$ & $\begin{array}{c}4.16 \\
(2.02)\end{array}$ & $\begin{array}{c}4.90 \\
(2.21)\end{array}$ & $\begin{array}{c}4.53 \\
(2.12)\end{array}$ & $\begin{array}{c}8.39 \\
(2.90)\end{array}$ & $\begin{array}{l}7.91 \\
(2.81)\end{array}$ & $\begin{array}{c}8.15 \\
(2.85)\end{array}$ & $\begin{array}{c}5.31 \\
(2.30)\end{array}$ & $\begin{array}{c}4.74 \\
(2.18)\end{array}$ & $\begin{array}{c}5.02 \\
(2.24)\end{array}$ \\
\hline M8 & Untreated control & $\begin{array}{c}0.38 \\
(0.92)\end{array}$ & $\begin{array}{c}6.88 \\
(2.61)\end{array}$ & $\begin{array}{l}12.35 \\
(3.50)\end{array}$ & $\begin{array}{c}9.62 \\
(3.05)\end{array}$ & $\begin{array}{l}11.33 \\
(3.36)\end{array}$ & $\begin{array}{l}15.48 \\
(3.93)\end{array}$ & $\begin{array}{l}13.41 \\
(3.64)\end{array}$ & $\begin{array}{l}16.12 \\
(3.99)\end{array}$ & $\begin{array}{l}18.47 \\
(4.28)\end{array}$ & $\begin{array}{l}17.30 \\
(4.13)\end{array}$ & $\begin{array}{l}11.44 \\
(3.37)\end{array}$ & $\begin{array}{l}15.44 \\
(3.92)\end{array}$ & $\begin{array}{l}13.44 \\
(3.64)\end{array}$ \\
\hline & $\mathrm{SE}(\mathrm{m}) \pm$ & 0.06 & 0.10 & 0.08 & 0.07 & 0.11 & 0.10 & 0.09 & 0.17 & 0.13 & 0.14 & 0.09 & 0.09 & 0.09 \\
\hline & $\mathrm{CD}$ at $5 \%$ & NS & 0.30 & 0.26 & 0.21 & 0.35 & 0.32 & 0.27 & 0.51 & 0.41 & 0.41 & 0.28 & 0.28 & 0.26 \\
\hline & $\mathrm{CV} \%$ & 16.78 & 8.25 & 7.92 & 8.69 & 8.01 & 7.74 & 8.46 & 9.17 & 7.33 & 10.65 & 6.24 & 6.10 & 8.01 \\
\hline
\end{tabular}

N.B: Figures in parenthesis are square root transformation 
Of these, treatments, module M4 was observed to be statistically at par with M2 (7.21 LH /leaf) and M3 (7.36 LH /leaf) whereas, the module M1 had noticed the population of leafhopper (9.51/leaf) and superior over module M8. The unprotected module M8 had observed the maximum leafhopper population (15.48 LH /leaf). At 14 days after spray the minimum population of leaf hopper was noticed in the treatment module M6 (7.65/leaf), and it was followed by M5 (7.78LH/leaf), M7 (7.91LH/leaf), M4 (9.41LH/leaf), M3 (9.79LH/leaf) and M2 $(9.86 \mathrm{LH} /$ leaf) and all these treatments were found to be statistically at par among themselves. The treatment module M1 had registered the leaf hopper population to the tune of (11.22/leaf) which in turn was at par with module M4, M3 and M2.The highest population of leafhopper was observed in module M8 (18.47LH/leaf).

The cumulative data on leaf hopper population as influenced by the various modules are presented the minimum population of leaf hopper was observed in module M6 (4.57/leaf), followed by module M7 (4.74/leaf) and module M5 (5.19/leaf) and these treatments were found to be statistically at par among themselves and module M6 was significantly superior over remaining treatment. Module M4 (6.04 /leaf) which was in turn found statistically as effective as M7 and M5. However, it was further statistically at par with M3 (6.66LH/leaf) and M2 (6.81LH/leaf), whereas, the treatment M1 was observed comparatively least effective (8.34LH/leaf) and was found statistically similar with module M2. The unprotected module M8 had registered the highest population of leaf hopper (15.44/leaf).

The data presented in Table 3 showed that pooled mean data on the incidence of aphid 24 hours before first spray were found statistically non-significant. However, incidence of leaf hopper in various treatments was found in the range of 0.08 leaf hopper /leaf in module M6 to 0.38 leaf hopper/leaf in module M8. However, other sucking pests thrips, whiteflies and mites were not initiated during both the years under study. The leaf hopper at 3 days after spray minimum population of leaf hopper was observed to the tune of 2.39/leaf in module M7 followed by module M6 (2.45 LH/leaf) and both the treatments were at par with each other and appeared to be statistically superior over other remaining treatments. The treatments module M5, M4, M3 and M2 accounted the leafhopper population in order of merit to the tune of $3.15, \quad 3.61, \quad 3.69$ and 3.95/leafhopper/leaf respectively, and these treatments were found to be at par among themselves and superior to M1 and M8. The treatment module $\mathrm{M} 1$ had registered the comparatively highest leafhopper population to the extent of 4.73/leaf and was found to be statistically similar with module M2. The unprotected module (M8) had recorded the highest incidence of leafhopper (9.62/leaf). Mean Leaf hopper population at 7 days after spray the treatment module M6 had shown the lowest leaf hopper population (4.50/leaf) and it was followed by module M7 (4.53LH/leaf) and module M5 (5.49 LH /leaf) and these treatments were statistically at par with each other. Module M6 and Module M7 were significantly superior over rest of the treatments except module M5 which was further found to be statistically at par with M4 (5.96 LH /leaf), M3 (6.46 LH /leaf) and M2 (6.62 LH /leaf). The treatment module M1 (8.71 LH /leaf) had noticed comparatively highest leafhopper population to the tune of (8.71 /leaf) showing comparatively less effective against leafhopper and superior to M8.The unprotected module had shown the highest population of leaf hopper (13.41/leaf). Mean Leaf hopper population at 14 days after spray the minimum population of leaf hopper was recorded in module M6 (7.52/leaf), 
followed by M5 (7.82LH/leaf), M7 (8.15LH/leaf) and M2 (9.51LH/leaf) and these treatments were found to be statistically at par among themselves. Of these module M6 and module M5 were significantly superior over rest of the treatments except module M7 and M2 whereas, further module M7 and module M2 were found to be statistically at par with M4 (10.12LH/leaf) and M3(10.54LH/leaf) and superior over M1 and M8. The treatments module M1 had registered the leaf hopper population to the extent of 11.13/leaf and it was also found statistically similar effective with the M2, M4 and M3 and superior over M8. The highest population of leaf hopper was noticed in the unprotected treatment module M8 (17.30/leaf). The data on pooled cumulative mean population of leaf hopper as influenced by the various modules presented in Table 3 the lowest population of leaf hopper (4.82/leaf) was recorded in module M6 followed by M7 (5.02LH/leaf) and M5 $(5.49 \mathrm{LH} / \mathrm{leaf})$ and they were statistically at par with other. Module M6 and M7 were significantly superior over remaining treatments except module M5 which was further found to be statistically at par with M4 (6.57LH/leaf) and M2 (6.69LH/leaf) and superior over M3, M1 and M8. Treatment module M3 had accounted the leafhopper population to the tune of 6.90/leaf and was statistically similar effective with M4 and M2. However, it was further statistically at par with M1 which registered the leaf hopper population to the tune of 8.19/leaf. The highest population of leaf hopper was accounted in unprotected module M8 (13.44/leaf). Presents findings are in agreement with Rajashekhar et al., (2010) who reported that the module comprising of seed treatment with imidacloprid @ $3 \mathrm{~g} / \mathrm{kg}$ seed + yellow sticky trap @ 25/ha and need base application of fipronil and neem oil effectively reduced the population of aphid, Jassid and WF in okra. Mandal et al., (2007) reported that soil application of neem cake along with three spray of neem oil and endosulfan gave maximum protection of jassids. Kumar and Singh (2001) reported from Bangalore (Karnataka) that seed treatment with imidacloprid (Gaucho 600 FS) at $12 \mathrm{ml} \mathrm{kg}-1$ was efficient in reducing leafhopper infestation with no phyto-toxic effect on okra plants Kaur (2002) who reported the superior bio-efficacy of ST with Imidacloprid @ 5g/kg and foliar spray of monocrotophos and cypermethrin in obtaining lowest population of cotton jassids. Kalyan et al., (2012) also reported that Spinosad, imidacloprid, acephate and fipronil effectively control the population of jassids and whiteflies in cotton. Above findings are aiso supported by Bagade and Ambekar, (2010) who also reported the superior efficacy of ST with Imidacloprid followed by cypermethrin $0.01 \%$ against aphid and jassids. Results are also in confirmation with Mishra (2002a) who reported that imidacloprid and thiamethoxam each at $25 \mathrm{~g}$./ha were significantly superior in controllong aphids and jassids followed by dimethoate @ 300 g.a.i. per ha and cypermethrin 100 g.a.i. per ha.

It is clear from the above findings that Module M6, M7 and M5 were found significantly most effective in minimizing the population of leaf hopper at 3,7 and 14 DAS. During summer and kharif 2015-16 seasons. While, Module M4, M2 and M3 were found to be moderately and statistically equally effective in minimizing the population of leaf hopper. Module M1 was found to be least effective. The treated module M6, M7 and M5 were found significantly most effective in reducing the population of leaf hopper due to the cumulative effect of seed treatment with imidacloprid, soil application of neem cake, maximum catches of leafhopper in yellow sticky trap and sequential spraying of synthetic pyrethorids (particularly cypermethrin, fenpopathrin and lambd- 
cyhalothrin) and systematic poisons (triazophos, acephate, quinalphos and thiamethoxam).

\section{References}

Anonymous, 2013. www.indiastat.com Anonymous. 2012 www.nhb.gov.in

Anwar, F., U. Rashid, M. Ashraf and M. Nadeem, 2010. Okra (Hibiscus esculentus) seed oil for biodiesel production. Applied Energy, 87(3), 779785.

Ashok Kumar, C. T. and C. Shivaraju, 2009. Evaluation of newer insecticide molecules against pod borers of black gram. Karnataka J. Agric. Sci., 22(3): 521-523.

Aykroud, W.R., 1963, I.C.M.R. Special Report series No. 42.

Bagade, A. S. and J. S. Ambekar, 2010. Imidacloprid, the potential pesticide against sucking pests of okra. J. Maharashtra Agril. Uni, 35 (1): 116118.

Baloch, A. F., S. M. Qayyum and M. A. Baloch 1990. Gomal Uni. J. of Res, 10, 191.

Kalyan, R. K., D. P Saini, P Urmila, P. Jambhulkar and A. Pareek, 2012. Comparative bioefficacy of some new molecules against jassids and whitefly in cotton. The Bioscan. 7(4): 641-643.
Kaur, S. 2002. Management of cotton jassid, Amrasca devastans (Dist.) and spotted bollworm, Earias sp. in Okra. Veg. Sci., 29: 172-175

Krishnaiah, K. 1980. Methodology for assessing crop losses due to pests of vegetable. Assessment of crop losses due to pests and diseases. Proceeding of workshops, held from Sept.19-30, 1977 at University of Agri. Sci, Bangalore, pp: 259-267.

Kumar, M. and A. K. Singh, 2001. Bioefficacy of insecticides against the cotton jassid, Amrasca biguttula biguttula (Ishida), on okra. Pest Manag and Econ.Zool. 9 (1): 55-58.

Mandal, S.K., S.B. Sah and S.C. Gupta, 2007. Management of insect pests on okra with biopesticides and chemicals. Ann. Pl. Protec. Sci., 15: 87-91.

Mane, S.A., U.M. Waghmare and G.A. Yadav, 2010. Bioefficacy and economics of some newer insecticides against fruit borer (Eariasvittella Fab.) of okra. Pestology. 34(5):39-42.

Rajashekhar, K., C Charanjeevi and T. Ratnasudhakar, 2010. Evaluation of different packages of integrated pest management for the management of okra pest complex. J. Res. Angrau., 38 (3-4): 9-14.

\section{How to cite this article:}

Vandana D. Mohod, A.Y. Thakare, A.V. Kolhe and Undirwade, D.B. 2019. Effect of Various Modules against Leaf Hopper, Amrasca biguttula biguttula Ishida on Okra. Int.J.Curr.Microbiol.App.Sci. 8(07): 1389-1398. doi: https://doi.org/10.20546/ijcmas.2019.807.166 